\title{
Design of automated collection and transfer robot for powder and flexible materials
}

\author{
Yibo Liu, Ying Pan* \\ College of Mechanical \& Automotive Engineering, Shanghai University of Engineering Science, Shanghai, China
}

\begin{abstract}
In order to solve the problem of collection and transport of toxic or odorous powder and flexible materials in industrial production, this article designs a robot that automatically collects and transports materials. Firstly, the system composition of the automated collection and transfer robot with the functions of collecting, transporting and trajectory traveling is discussed. The working principle of each functional module is introduced. The mechanical structure design of parallel parallelogram switch mechanism, grade I and II height adjusting mechanism, transfer device and walking device is completed. Secondly, a control system with STM32 chip as the core is designed. With the signal of relevant sensors as the auxiliary, the material collection, posture adjustment and trajectory traveling are realized. Finally, the rationality of the scheme is verified by the static and dynamic simulation of the key components of each functional module of the robot. The designed robot had been proved to have good running stability and reliability by simulation results, prototype trial and commissioning for key parts.
\end{abstract}

\section{Introduction}

China's industrial development is relatively late. In chemical, pharmaceutical and other industrial production, toxic or odorous powder and flexible materials often appear as products or waste. The collection and transporting of these materials are basically done by manpower, and the labor intensity of workers is high and the working environment is not conducive to health $[1,2]$. Undoubtedly, the application of automation technology to the collection and transporting of powder and flexible materials will effectively solve this problem and improve the working environment of workers. The research on this issue has gradually attracted the attention of relevant enterprises, scientific research institutions [3 6].

Powder and flexible materials are usually collected manually and carried by carts or forklift trucks after packing or bagging. This type of operation has certain safety hazards and the working environment is harmful to the field workers [7]. If the material needs to be filled by weight, the worker needs to weigh the material again, which is inefficient.

Under the above background, this article designs an automated collection and transfer robot for powder and flexible materials. Based on the wheeled robot, the robot adds material receiving mechanism, collecting device, transfer device and walking device, and is equipped with sensors and control systems to realize automatic collection and transporting of powder and flexible materials. Through systematic movement of various mechanisms, robots can achieve higher efficiency material collection and reduce the lifting and splashing of powder and flexible materials. The robot receiving bucket is equipped with a gravity sensor, which can realize quantitative collection of materials; the robot can also transport the collected materials to a designated position according to the predetermined trajectory through the walking device [8].

\section{Overall layouts}

As shown in Fig.1, the application scenario of an automated collection and transfer robot is shown. The robot moves to the lower part of the conveyor belt, adjusts the posture, the switch mechanism is opened, and the I and II height adjustment mechanisms adjust the distance from the conveyor belt to realize the collection of material.

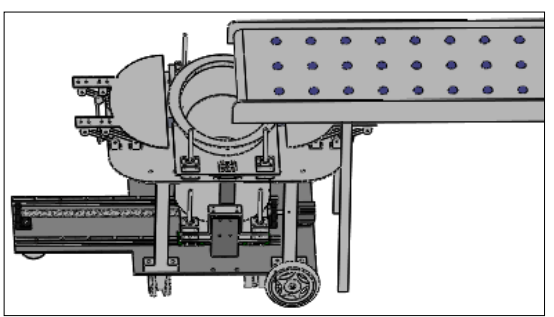

Figure 1.application scenario

\section{The composition of automated collection and transfer robot}

*Corresponding author: ap_xjtu@163.com 
The designed automated collection and transfer robot for powder and flexible materials is mainly composed of wheeled robot body, parallel parallelogram switch mechanism, I-level height adjusting mechanism, II-level height adjusting mechanism, transfer device, posture adjusting, walking device, sensor and STM32-based control system. The overall mechanism of the robot is shown in Fig.2.

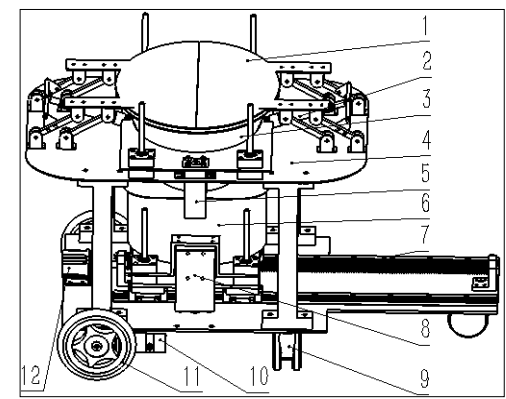

1.Switch baffle 2 .MA16 cylinder 3. Collection port 4. Robot body 5 . SAD20 cylinder 6 . Receiving bucket 7 . Screw 8 . MGPM16 cylinder 9. Universal wheel 10. Walking motor 11. Walking wheel 12. Stepper motor

Figure 2. Automated collection and transfer robot

The wheeled robot adopts the differential speed of the two driving rear wheels to achieve a predetermined trajectory. The switch mechanism can be opened and closed as needed. It should be opened when collecting powder and flexible materials, while closed in transfer process to prevent material from flying or splashing. The I-level height adjusting mechanism can adjust the distance between the collection port and the conveyor belt, and the II-level height adjusting mechanism can adjust the distance between the receiving bucket and the collection port to realize the sealing of the material collection process. The transporting device can transport the II-level adjusting mechanism including the receiving bucket to the edge of the robot, so as to facilitate the material removal in the subsequent process.

\subsection{Design of parallel parallelogram switch mechanism}

In industrial production, easy-to-raise powder and flexible materials with pungent odors often appear as products or waste. In order to solve the problem of human and environmental hazards caused by the lifting of materials and the irritating gases generated during transportation, we designed a parallel parallelogram switch device.

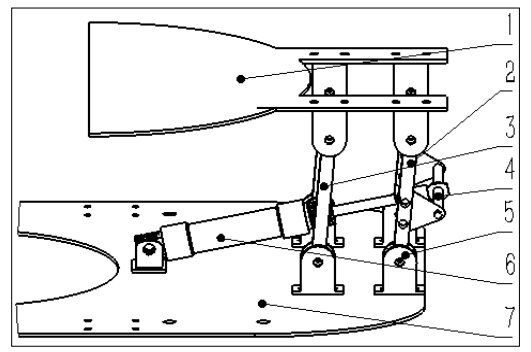

1. Switch baffle 2. Main rotating rod 3. Sub-rotating rod 4.Cylinder connecting rod 5. Hinging 6. MA16 cylinder 7. upport plate

Figure 3. Parallelogram Mechanism

As shown in Fig. 3, the driving original is a cylinder, and the telescopic movement of the cylinder drives the cylinder link to rotate around the hinge, thereby driving the parallel parallelogram mechanism to realize the opening and closing action of the switch baffle. The trajectory of the switch mechanism affects the size of collection port and the distance between collection port and the conveyor belt. The kinematic analysis of the parallelogram mechanism is carried out.

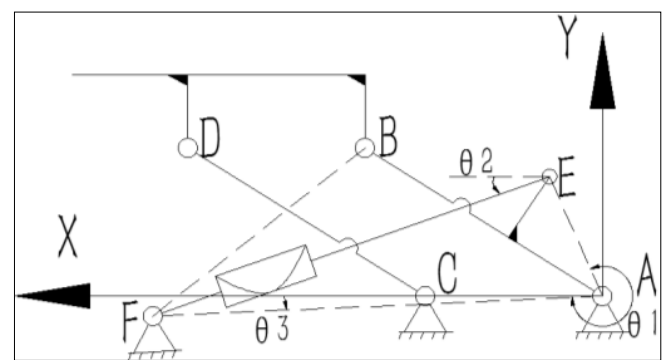

$\mathrm{L}_{\mathrm{AB}}=\mathrm{LCD}=78 ; \mathrm{L}_{\mathrm{AC}}=\mathrm{L}_{\mathrm{BD}}=48 ; \mathrm{LAE}_{\mathrm{AE}}=35.64 ; \mathrm{L}_{\mathrm{AF}}=99.25 ; \theta 3=4.6^{\circ}$

Figure 4. Mechanism Diagram

The movable member $n=5$, the low sub $P_{L}=7$, the high sub $\mathrm{P}_{\mathrm{H}}=0$, and the mechanism freedom $\mathrm{F}=3 \mathrm{n}-2 \mathrm{P}_{\mathrm{L}}-\mathrm{P}_{\mathrm{H}}=1$, the mechanism has a certain motion [9]. The movement of the switch baffle depends on the rod AB. Next, the rod $\mathrm{AB}$ is analysed and calculated.

From the vector equation $\overrightarrow{A E}+\overrightarrow{E F}=\overrightarrow{A F}$, the complex equation

$$
L_{A E} e^{i \theta_{1}}+L_{E F} e^{i \theta_{2}}=L_{A F} e^{i \theta_{3}}
$$

According to the Euler formula, and the real and imaginary parts are taken separately, the equation is obtained:

$$
\begin{aligned}
& L_{A E} \cos \theta_{1}+L_{E F} \cos \theta_{2}=L_{A F} \cos \theta_{3} \\
& L_{A E} \sin \theta_{1}+L_{E F} \sin \theta_{2}=L_{A F} \sin \theta_{3}
\end{aligned}
$$

$\mathrm{L}_{\mathrm{EF}}=114+\mathrm{vt}$; $\mathrm{v}$ is the cylinder speed, $\mathrm{t}$ is the running time Obtained by the above equation:

$$
\theta_{1}=2 \operatorname{arctg} \frac{565.96+\sqrt{50069492.68-\left((114+\mathrm{vt})^{2}-11120.77\right)^{2}}}{4067.46-(114+\mathrm{vt})^{2}}
$$

Deriving equation (1) for time

$$
\begin{aligned}
& L_{A E} \omega_{1} i e^{i \theta_{1}}+L_{E F} \omega_{2} i e^{i \theta_{2}}=L_{A F} \omega_{3} i e^{i \theta_{3}} \\
& \omega_{3}=\omega_{1} \frac{35.64 \sin \left(\theta_{1}-\theta_{2}\right)}{99.25 \sin \left(4.6^{\circ}-\theta_{2}\right)} \\
& \omega_{2}=-\omega_{1} \frac{35.64 \sin \left(\theta_{1}-4.6^{\circ}\right)}{(114+v t) \sin \left(\theta_{2}-4.6^{\circ}\right)}
\end{aligned}
$$

In the mechanism diagram shown in Fig.4, the movement law of the switch baffle is determined by the main rotating rod $\mathrm{AB}$, and the rod $\mathrm{AB}$ and the $\mathrm{AE}$ have the same motion law. That is to say, after determining the speed of the electric cylinder, the angular displacement 
and angular velocity of the rod $\mathrm{AB}$ and the switch port can be obtained.

\subsection{Design of I-level height adjustment mechanism}

The I-level height adjustment mechanism is used to adjust the distance between the collection port and the conveyor belt, reduce the spillage of powder and flexible materials, and improve the collection rate of materials. The structure is shown in Fig.5.

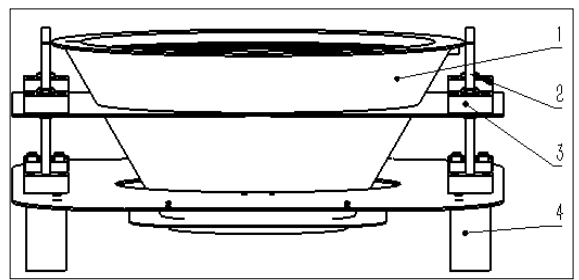

1. Collection port 2. Optical axis 3. Linear bearing fixing 4. SAD20 cylinder

Figure 5. I-level height adjustment mechanism

In order to increase the collection rate of powder and flexible materials, the collection port adopts a taper design with large opening degree, which can increase the contact area between collection port and the powder and the flexible material. The collection port is powered by the cylinder movement and guided by the optical axis, and the distance from the conveyor belt can be adjusted within a limited stroke. The bottom of collection port is designed with a baffle design, and the surface is covered with a layer of rubber to achieve sealing with the receiving barrel.

\subsection{Design of II-level height adjustment mechanism and transfer mechanism}

The II-level height adjustment mechanism is used to adjust the distance between the receiving bucket and the I-level height adjustment mechanism, to ensure the sealing of the two-stage mechanism, and to reduce the lifting and splashing of powder and flexible materials. The transfer mechanism is used to transport the receiving bucket of the storage material and the II-level adjustment mechanism to the edge of the robot, and the material is recycled or packaged by the robot or human. The II-level height adjustment mechanism and the transfer mechanism are shown in Fig.6.

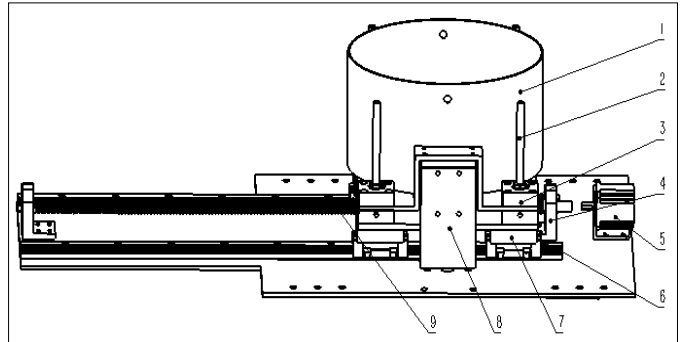

1. Receiving bucket 2. Optical axis 3. Linear bearing fixing parts 4. Screw base 5. Motor 6. Guide rail 7. Slider 8. MGPM16 cylinder 9. Lead screw

Figure 6. II-level height adjustment mechanism and transfer mechanism
The transfer mechanism is a guide screw mechanism, the motor drives the lead screw to rotate, and the guide rail guides to transport the material to the edge of the robot. A gravity sensor is installed at the bottom of the receiving bucket to measure data in real time and transfer the data to the STM32 for processing. When the weight reaches the specified requirements, the II-level height adjustment mechanism is reset and the transfer device transports the material out.

\subsection{Posture adjustment and walking device}

The connection between collection port and the conveyor is affected by the layout and the environment. The posture adjustment mechanism aims to make adaptive adjustments to the above factors to ensure the efficiency of collecting materials [10]. The walking device enables the transfer robot to follow the prescribed track and transport the collected materials to the designated location.

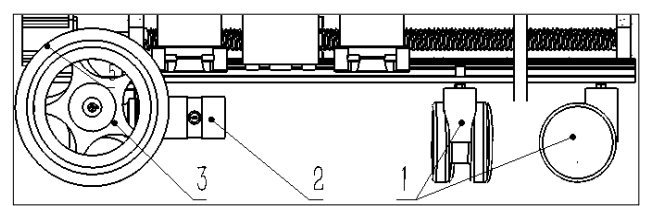

1.Universal wheel 2. Walking motor 3. Walking wheel

Figure 7 . Posture adjustment and Walking device

\section{Control system}

The control system is used to control the operation of the automated collection and transfer robot. The power components in the robot are cylinders and motors and the main chip uses the current mainstream STM32 chip. The action of each mechanism is realized by programming, and the relevant parameters can be set on the parameter panel of the body. A variety of sensors are installed on the body. During the running process, the data is collected in real time and transmitted to the processor. The processor analyzes the signal and makes adaptive processing according to different situations to ensure the normal operation of the robot.

Before starting material collection, the quality of the collected materials is set through the parameter board. This data is used to compare the sensor feedback data to determine whether the specified weight is reached. The parameters of each organization's operation have been set in the chip. After the initialization is completed, the robot can realize repeated collection and transfer of the same material.

\section{Simulation analysis, prototype trial and commissioning}

There are series and parallel kinematic chains in the various mechanisms of the automated collection and transfer robot. There are rotating pairs and moving pairs in these kinematic chains, and the reliability of each moving pair affects the stability of the entire system. Therefore, the mechanism in the system is simulated, 
analyzed and trial-manufactured to verify the feasibility and rationality of the mechanism design scheme.

\subsection{Simulation analysis of the switch mechanism}

In order to verify the rationality of the switch mechanism, SOLIDWORKS Motion is used to simulate the dynamics simulation. By analysing the cylinder force curve, the angular velocity curve of the switch mechanism and other indicators to intuitively analyze the operating state of the mechanism. The model of the organization is imported into SOLIDWORKS Motion and the corresponding parameters are configured.

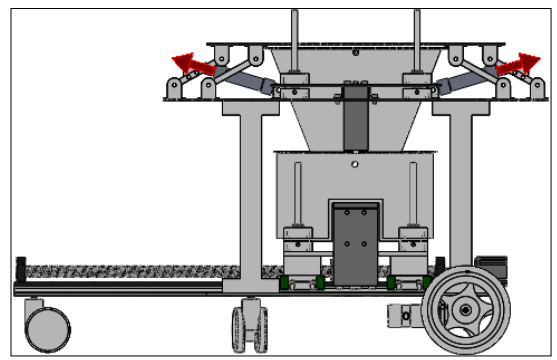

Figure 8. Motion configuration

During the analysis, the parts are matched according to the actual working conditions, and the linear damping and torsional damping parameters are set. The linear motor is configured according to the cylinder actual designed operating speed of $20 \mathrm{~mm} / \mathrm{s}$, and the motion calculation is run to calculate the result and generate a diagram.

The cylinder connecting rod is a key component connecting the cylinder and the parallel parallelogram mechanism, and its deformation failure will affect the operation of the entire parallelogram switch mechanism. Firstly, the force of the cylinder during the whole operation is analyzed, and the bearing capacity of the selected cylinder is verified. Secondly, according to the maximum force of the cylinder, the finite element analysis of the cylinder connecting rod is carried out to test the stress magnitude and deformation amount. Finally, the variation law of the angular velocity of the switch mechanism is analyzed to verify the rationality of the whole mechanism.

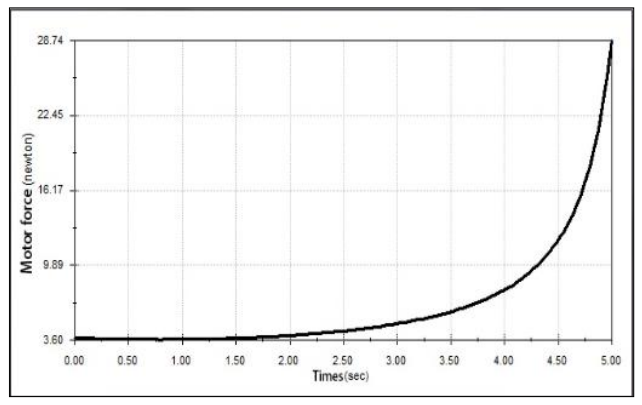

Figure 9.Cylinder force curve

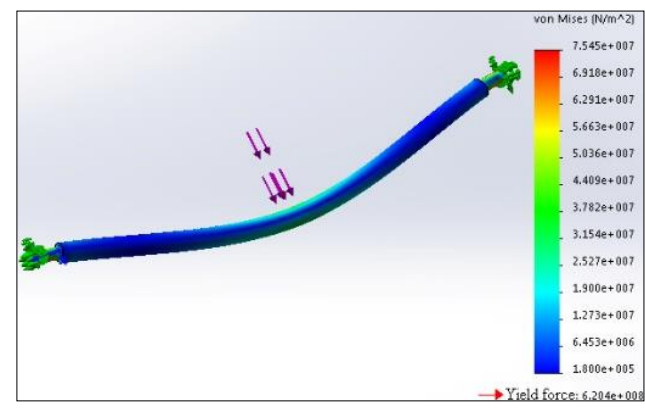

Figure 10. Static analysis

It can be seen from Fig. 9 that the maximum force of the cylinder during the entire operation is not more than $30 \mathrm{~N}$. The cylinder capacity is about $50 \mathrm{~N}$, so the cylinder can meet the design requirements. The cylinder connecting rod is the key connecting rod of the switch mechanism, and its material is Q235. The cylinder force acts directly on the cylinder connecting rod, and static analysis by SOLIDWORKS Simulation to judge its deformation. The result is shown in Fig. 10.

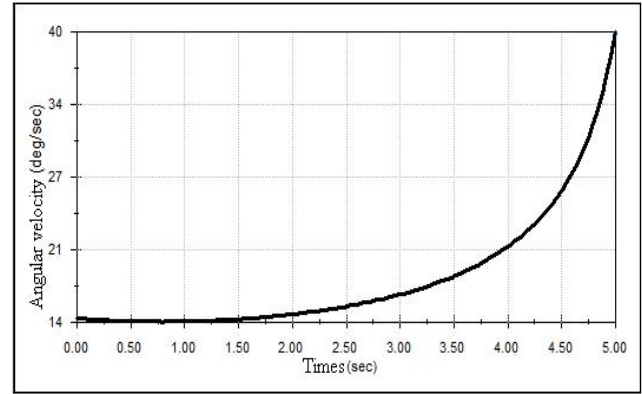

Figure 11.angular velocity analysis

The static analysis shown in Fig.10 shows that the stress on the cylinder link is much less than the yield force of the material, so there is no large deformation throughout the operation. In the angular velocity analysis shown in Fig.11, the angular velocity curve changes gently, and no abrupt changes and breakpoints occur, that is, the switching process is continuous. Through the above analysis, the rationality and feasibility of the design scheme of the switch mechanism are determined.

The simulation analysis of the mechanical structure of other modules is similar to that of the switch mechanism, which is not be repeated here.

\section{Conclusion}

The automated collection and transfer robot for powder and flexible materials designed and developed in this study adopts the modular design idea. Sensors and control systems are used in the robot to achieve quantitative collection of powder and flexible materials. The mechanical structure of the collection process is closed, thus reducing the splash of powder and flexible materials. Moreover, the robot can transport the collected material to a designated position according to a predetermined trajectory. The results of the prototype commissioning show that the robot can achieve higher efficiency material collection. It can improve the working 
environment of workers, and the automation level of industrial production. Hence, it has high practical industrial application value.

\section{References}

1. Sanjiang Li, Yana Li. The status quo and development trend of foreign packaging machinery and the development strategy of domestic packaging machinery $[\mathrm{J}]$. Science \& Technology Information, (2015), 13 (35): 154-156.

2. Tianran Wang Editor. Robot [M]. Beijing: Chemical Industry Press.( 2002).

3. Zhenyu Zhang, Wushan Cheng. Design of Selfadaptive sealing machine for silt and dump material packaging line[J]. Light Industry Machinery,(2017), 35(06): 62-67.

4. Shuang Wu. Mechanical Design and Development on Automatic Fruit-vegetable Packing Machine [D]. Zhejiang agriculture and Forestry University, (2014).

5. Longfei Pan, Weidi Liu, Yang Yang, et al.Fluid analysis and optimization of waste bucket in highspeed automatic stamping production line for airconditioner fin based on NX. China Metalforming Equipment\&ManufacturingTechnology,(2016),51(05 ):21-24.

6. Yunfei Song. Material transport systems of coal sampler analysisand design optimization[D]. Xi'an University of Architecture and Technology, (2014).

7. Ronggui Dao, Jinwu Zhang, Xiang Yang. Modeling and Analysis of Logistics System for Recycling Waste from Cigarette Production Line[J]. Tobacco Science \& Technology, (2010)(12): 16-20.

8. Wei Zhang, Yixiong $\mathrm{Wu}$, Xin Jin.Trajectory Planning of Posture Adjustment of Welding Mobile Robot During Auto-searching Weld Line. Chinese Journal of Mechanical Engineering,(2005)(05):215220.

9. Wenwei Zheng, Kejian Wu editor. Mechanical Principles [M]. Beijing: Higher Education Press.( 1956).

10. Zixing Cai editor. Robotics [M]. Beijing: Tsinghua University Press. (2000). 